\title{
Changes in the Content of Nucleic Acids in Brain Neurons under Various Modes of Low Radiation Exposure
}

\author{
Ushakov IB*, Fedorov VP, Komarevtsev VN and Dyachkov AA \\ Federal Medical Biophysical Centre (FMBC), Russia \\ *Corresponding author: Igor Borisovich Ushakov, Burnasyan Federal Medical Biophysical Centre (FMBC), Moscow, Russia \\ To Cite This Article: Ushakov IB, Fedorov VP, Komarevtsev VN, Dyachkov AA. Changes in the Content of Nucleic Acids in Brain Neurons \\ under Various Modes of Low Radiation Exposure. 2020 - 9(2). AJBSR.MS.ID.001369. DOI: 10.34297/AJBSR.2020.09.001369.
}

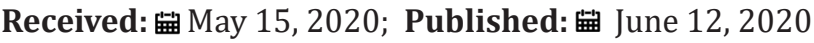

\section{Minireview}

Nucleic acids are the most important radiation target of cells. However, despite the huge number of researches on the problem, their dynamics in the brain neurons throughout the entire period of post radiation remains unexplored. In this regard, the aim of this research was to study the state of nucleic acids in the brain neurons of rats after irradiation at doses of 0.1 to 1 Gy over their entire life span. The experiment was performed in compliance with the rules of bioethics on 390 white male mongrel rats at the age of 4 months. by the beginning of the study, weighing $210 \pm 10 \mathrm{~g}$, which were irradiated with gamma quanta (Co-60) with an energy of 1.2 $\mathrm{MeV}$ once and fractionated (in equal portions for 5 days) at total doses of $0.1 ; 0.2 ; 0.5$ and 1.0 Gy with a dose rate of $0.5 \mathrm{~Gy} / \mathrm{h}$ and 0.5 Gy with a dose rate of $0.5 ; 1.0 ; 2.5$ and $6.6 \mathrm{~Gy} / \mathrm{h}$. The animals that were exposed to false irradiation - at the same time as the irradiated ones - served as a control. Different parts of the brain were collected after 1 day, 6, 12, 18 and 24 months during postexposure period? The study was carried out for the full life span of the animals. Each group had adequate age control. After standard histological procedures, nucleic acids (DNA in nuclei and RNA in cytoplasm and nucleoli) of neurons were detected on paraffin and cryostat sections stained by S. Shea (1970) with appropriate control of RNase and DNase. Neuromorphological methods were used to evaluate the morphometric and tinctorial parameters of neurons, as well as the dynamics of nucleic acids in neurons throughout the life span of animals. Statistical processing of the results was carried out using software packages Statistica 6.1 using parametric criteria, mathematical modeling and determination of the forecast of their development.
Studies have shown that both control and irradiated animals throughout their lives experienced wave-like changes in the content of nucleic acids in the brain neurons with a gradual decrease by the end of the irradiation period. Meanwhile, changes in DNA nuclei and RNA in nucleoli are usually associated with changes in their size, and changes in cytoplasmic RNA, apparently, are associated with the physiological state of neurons (rest, excitation, inhibition) and the corresponding structural and functional rearrangement of nerve cells. Regression analysis showed that irradiation in studied doses and exposure modes has a nonlinear stochastic effect on studied neuromorphological parameters, has no dosetime dependence and does not cause significant organic changes in the neurons of the brain. At the end of the irradiation period, when the death of both irradiated and control animals is observed, the content of nucleic acids in neurons is statistically significantly reduced in all studied groups, and mainly in irradiated animals. At the same time, the dependence on the radiation dose and exposure mode is not established, but with an increase in the dose rate, changes in the content of both DNA and RNA in the brain neurons become more polymorphic. The most polymorphic changes in the content of nucleic acids is observed in the pear-shaped neurons of the cerebellum cortex, and the parietal cortex neurons were more resistant to these indicators.

The research has shown that at all doses and modes of radiation exposure throughout the radiation period, there are wave-like changes in the content of cytoplasmic and nucleolar RNA, as well as nuclear DNA in brain neurons without significant changes in their indicators in relation to age control. The dynamics of changes 
in the content of DNA and RNA in neurons under radiation effects fluctuates around the norm, usually within $10-20 \%$, and rather has the character of random fluctuations. At the same time, in some cases, changes in the content of nucleic acids are borderline, when it is no longer the norm, but not yet a pathology. It is likely that with an increase in the dose of radiation or the concomitant action of harmful and dangerous environmental factors, changes in the content of nucleic acids in the neurons of the brain will become more pronounced and unidirectional.

Thus, any significant radiation-induced changes in the content and topochemistry of histochemical reactions products during the detection of nucleic acids in the structures of brain neurons were not revealed. However, in certain periods of observation, the content of nucleic neurons acids in irradiated animals changes more significant than in animals of age control.

Unfortunately, experiments with modifications of nucleic acids of brain neurons throughout life after irradiation at doses that do not cause deterministic effects have not been conducted before and it is not possible to compare our data with other studies. 